\title{
Managing Sexually Inappropriate Behaviour among the Older Adults with Dementia
}

\author{
Rachelle Tan-Patanao ${ }^{1}$, Eden XJ Tay ${ }^{2}$, WT Chang ${ }^{3}$, SC Lim ${ }^{4 *}$ \\ ${ }^{1}$ Resident Physician, Department of Geriatric Medicine, Changi General Hospital, Singapore. \\ ${ }^{2}$ Family Medicine Resident, MOH Holdings (MOHH), Singapore. \\ ${ }^{3}$ Principal Clinical Pharmacist, Department of Pharmacy, Ng Teng Fong General Hospital, Singpore. \\ ${ }^{4}$ Adjunct Associate Professor, Senior Consultant, Department of Geriatric Medicine, Changi General Hospital, \\ Singapore. \\ lim.si.ching@singhealth.com.sg \\ *Corresponding Author: Si Ching Lim (MB.ChB, MRCP), Adjunct Associate Professor, Senior Consultant, \\ Department of Geriatric Medicine, Changi General Hospital, Singapore.
}

\section{Abstract}

Neuropsychiatric symptoms of dementia are more distressing for the caregivers of persons with dementia, compared to the cognitive symptoms. Inappropriate sexual behavior or hypersexuality is one of the behavioral problems which cause significant caregiver stress and without proper management, may result in institutionalization and social isolation. There is currently no official treatment guideline in the management of inappropriate sexual behavior in dementia. Successful management of sexual disinhibition is often multifactorial and the approach should be individualized with non-pharmacological and pharmacological approach.

Keywords: dementia, BPSD, hypersexuality, sexual disinhibition, inappropriate sexual behavior (ISB)

\section{INTRODUCTION}

The dementia syndrome is an umbrella of neurodegenerative disorders which progressively impacts on the person's cognition, resulting in emergence of behavioural symptoms and decline in their abilities to carry out their routine activities of daily living. Behavioral and psychological symptoms of dementia are common among the persons with dementia, the symptom spectrum includes agitation, aberrant motor behavior, anxiety, elation, irritability, depression, apathy, disinhibition, delusions, hallucinations, and sleep or appetite changes. A crosssectional study by Lyketsos CG et al, estimated the prevalence of the neuropsychiatric symptoms of dementia is about $50 \%$ to $80 \%$ among persons with dementia throughout the course of the disease. ${ }^{[1,2,3]}$ Based on a local study done in 2013, the prevalence of BPSD amongst the study population with dementia was 67.9 percent. ${ }^{[4]}$

The most common BPSD reported in people with dementia are apathy, depression, anxiety and depression ${ }^{[1,5,6]}$ while inappropriate sexual behavior (ISB, also known as sexually disinhibited behavior, or hypersexuality) is estimated to be present in $2-17 \%$ of dementia patients. ${ }^{[7]}$ It is more commonly associated with vascular type of dementia, ${ }^{[8]}$ with higher prevalence in residents of skilled nursing facilities $(25 \%)$ and in those with severe dementia. ${ }^{[9,10]}$ ISB can be a threat to the mental and physical health of patients and others. ${ }^{[11]}$ It often results in increase care burden, ${ }^{[12]}$ feelings of anxiety, embarrassment or unease in the caregivers causing disruption in the continuity of care at home, ${ }^{[13]}$ hence leading to increased risk of institutionalization. ${ }^{[14]}$

Currently, there is no established treatment algorithm in the management of dementia related sexual disinhibition. Approach should be individualized which can either be non-pharmacological interventions, pharmacological interventions or a combination of both.

This paper illustrates two cases of sexually inappropriate behavior in an acute hospital setting, 
and their management strategies. A short review of non-pharmacological and pharmacological treatment methods is also summarized.

\section{Patient 1}

Mr. A is a 72 years old Malay Gentleman, widowed, with a background history of hypertension, hyperlipidemia, a previous right sided pontine infarct conservatively managed with single anti-platelet therapy (Aspirin $100 \mathrm{mg}$ OM), asymptomatic infrarenal abdominal aortic aneurysm and previous deep vein thrombosis of the left lower limb. He was community ambulant prior to admission, lives with his son and daughter with no designated full time caregiver.

He had recently been discharged from Rehabilitative Medicine for a short stint of rehabilitation after an open mesh repair for an inguinal hernia. During his stay, he was referred to Geriatric Medicine as they had incidentally noted possible cognitive impairment. About 2 months after his discharge, he was noted by his family to have developed behavioral changes at home with aberrant vocalisation of loud sounds and moans, which could last late into the night. His loud vocalisation would sometimes be accusatory in nature, where he accused his children of not taking good care of him despite his advanced age, and on occasions, escalated to physical agitation where he would bang his fists onto tables or hard surfaces. Thankfully this never translated to physical violence against his family members, and he was admitted for further workup of this subacute behavioral change.

During his inpatient stay, Mr. A exhibited symptoms of sexual disinhibition towards the nursing staff, being explicit and suggestive in both his choice of words and actions. His verbalisation increased in both volume and intensity, with increasing physical agitation as well, at times requiring physical restraints. The routine lab investigations were all unremarkable. Blood tests for HIV and VDRL serologies were negative. Magnetic Resonance Imaging (MRI) of his brain revealed moderate global cortical atrophy, with no acute infarct, hemorrhage or pathological leptomeningeal enhancement on a background of chronic vascular microinfarcts.

Further corroborative cognitive history revealed > 6 months history of short-term memory loss, with agnosia and executive dysfunction. His family also revealed that in the months prior to his previous admission, he had sporadic episodes of auditory hallucinations together with behavioral change. The diagnosis of probable mixed Vascular and Alzheimer's Dementia, likely Frontotemporal predominance with Behavioral and Psychotic Symptoms (BPSD) was most likely. The behavioral problems were causing distress to the caregivers, and he occasionally threatened ward nurses with assault. Various non-pharmacological strategies were tried but failed. Pharmacological management was initiated to manage his incessant demands for sexual acts.

These included:

- Fluvoxamine, Gabapentin and Finasteride, $\beta$ blocker were chosen. All these were chosen for their off-label indications of causing sexual dysfunction.

- Donepezil for component of probable Alzheimer Dementia and also for its libido lowering effects.

- Olanzapine for aggression, on as required basis.

His behavioral symptoms gradually improved, and his sexual disinhibition was kept under control with the above medications. He remained clinically well and stable on discharge 3 weeks later.

\section{Patient 2}

Mr. Y is a 91-year old gentleman with a background history of ischemic heart disease, hypertension, hyperlipidemia, chronic kidney disease and benign prostatic hypertrophy. He is pre-morbidly ADL independent and lives with two tenants. He has a fulltime caregiver who is a domestic worker. Mr. Y was admitted for syncope lasting for one minute, with no preceding symptoms. Physical examination was unremarkable. Neurological examination showed no evidence of localizing signs. His GCS was 15/15 throughout.

Initial investigations including CT brain were unremarkable, except for 24-holter which showed runs of supraventricular tachycardia (SVT) with no significant pauses. Cardiologists recommended $\beta$ blocker if needed but was not given, since he was asymptomatic during runs of SVT.

The syncopal episode was likely due to postural hypotension likely attributed to SVT and medications Alfuzosin and Perindropil. Perindopril was discontinued and Alfuzosin was switched to Tamsulosin. 
During his hospital stay, Mr. Y was noted to be agitated with sleep wake reversal. Cognitive and behavioral history from family was suggestive of dementia. His abbreviated mental test score was 4/10 and Chinese MMSE was $14 / 28$. Family reported that Mr. Y touched his female caregiver inappropriately at night, and if he could not sleep, he would wander into the maid's room. In view of this, the family had to change maid four times in eight months.

Mr. Y was started on Escitalopram, and Finasteride was added for BPH which since he had postural hypotension with $\alpha$ blocker. Finasteride also reduces libido which might be helpful in treating sexual disinhibition. Dementia education and counselling was done to the family and advised to hire a male helper if possible. Mr. Y behavior showed significant improvement, with improved sleep pattern and no further sexually disinhibited behavior noted. Mr. Y was discharged well and stable.

\section{Discussion}

Inappropriate sexual behavior has been defined differently by different authors across different studies. According to Fabà, J \& Villar, F, the definition proposed by Johnson et al. (2006) is more complete. ${ }^{[15]}$ Inappropriate sexual behavior (ISB) as defined by Johnson et al, "is a disruptive behavior characterized by a verbal or physical act of an explicit or perceived sexual nature, which is unacceptable within the social context in which it is carried out". [16] This definition has been expanded by de Medeiros et al, to distinguish between intimacy-seeking and disinhibited sexual behaviors. Intimacy-seeking behavior includes affectionate behaviors directed towards a wrong target (e.g., trying to kiss a stranger who the person with dementia mistook for his or her partner), while disinhibited sexual behavior includes impulsive, indiscriminative and invasive behaviors marked by the apparent loss of control and prompted by environmental stimuli such as close proximity of a potential sexual partner. Grabbing a caregiver's breasts during bathing or toileting routines would be a clear example of sexual disinhibition. ${ }^{[17]}$

Inappropriate sexual behavior (ISB) is common in people with dementia. The occurrence of ISB is reported to range from 7 to $25 \%$, with higher prevalence in residents of skilled nursing facilities and in persons with more severe cognitive impairment. [9, ${ }^{10]}$ Physical manifestations appear to be more frequent in males. ${ }^{[18]}$ Women seem to show more verbal disinhibition. ${ }^{[19]}$ In the context of dementia, ISB may be an attempt to seek emotional connection, love and relief for anxiety and insecurity. ${ }^{[20]}$ Bearing in mind, sexual needs are still present among the elderly, ${ }^{\left[{ }^{[0]}\right.}$ it is sometimes difficult to differentiate when physiological or emotional needs had breached the boundaries to become pathological. In an institution setting like nursing home and hospital, there is no privacy to allow for intimacy and sexual acts among the older residents are often frowned upon by society. ${ }^{[7]}$

ISB can be categorized into sex talk (using foul language), sexual acts (acts of touching, grabbing, exposing the genitals or masturbating), and implied sexual acts (openly reading/ watching pornographic material). ${ }^{[9]}$ Other authors gave more descriptive examples such as indecent exposure in public places, obscene sex language, public masturbation, touching others breasts or genitals and inappropriate propositions to others. ${ }^{[10,21,22]}$ Both Mr. A and Mr. Y presented with sexual talk and acts of touching and masturbating. Inappropriate sexual behavior can be distressing to formal or informal caregivers, and they may find it difficult to cope. ${ }^{[23]}$ Formal caregivers working in residential aged care facilities may cope better, since they are trained. However, conflicts may arise when the behaviors are directed towards them or the visitors. ${ }^{[24]}$ ISB poses significant negative impact for the persons with dementia, since their indiscretion can result in acquisition of sexually transmitted diseases, ${ }^{[25]}$ genital trauma, ${ }^{[26]}$ decline in the quality of the care received, institutionalization or becoming the target of mockery and rejection. ${ }^{[15]}$

ISB can be divided into conventional and non-paraphilic (sexual interest arising within socially and culturally accepted boundaries), versus unconventional and paraphilic (sexual arousal to atypical objects/ situations like inanimate objects, children, animals and non-consenting partner). ${ }^{[27]}$

Management of ISB in dementia should be tailored according to each individual presentation. To guide optimal management of ISB, the evaluation should include a thorough medical history and physical examination and targeted laboratory testing. [7] History should attempt to elicit depressive mood, attention-seeking behavior, substance use, psychosis, or long-standing hypersexual personality traits, as approach will be based differently. The history ask about precipitants like environmental or emotional 
triggers, medications (benzodiazepines, dopamine agonists, androgen supplements) or misinterpretation of nonsexual acts (routine nursing care) and consequences of the behavior.

Faba J, stated that dementia may cause a number of changes in the patient's social sphere that can lead to the development of ISB ${ }^{[15]}$ such as lack of a regular sexual partner, lack of privacy among institutionalized residents, moving to a new accommodation that is less familiar and stimulating. ${ }^{[7]}$

As the elderly are often taking multiple medications for their background medical problems, there are certain medications which may exacerbate sexually disinhibited behaviors. Benzodiazepines may be used to manage agitations, they may paradoxically aggravate disinhibition and agitation in older persons. Benzodiazepines bind to the Gamma-aminobutyric Acid (GABA) receptors in the brain, enhancing the physiologically inhibitory actions of the endogenous GABA in the central nervous system (CNS). In susceptible individuals, the inhibitory effects may predominate in the cortex leading to the dampening of the cortical restraining influence on antisocial impulses. In addition, benzodiazepines reduce ability to ensure appropriate social behaviors, by ignoring the environmental cues. ${ }^{[28]}$

Usage of Dopaminergic agent, like levodopa may directly trigger sexually disinhibited behavior by direct activation of dopamine receptors. [29] Androgenic agents like testosterone, are known to increase aggressiveness and impulsiveness. A study of nandrolone, an androgen, in rats showed sexual disinhibition by its effect on the serotonergic system in the prefrontal cortex and amygdala. ${ }^{[30]}$

Although there is no specific guideline in the management of ISB in dementia, the approach should be similar to other BPSD symptoms. The management strategies start with non-pharmacological measures. Review of medication is important as some medications may aggravate or worsen the disinhibition. Pharmacologic therapy should only be initiated if symptoms fail to respond to more conservative measures.

\section{Non-Pharmacological Management}

There is ageneral agreement that non-pharmacological approach should be the first-line treatment for ISB in elderly people with dementia, ${ }^{[13]}$ principally because of concerns about safety and ethics. ${ }^{[31]}$ It can be divided into environmental, behavioral (or cognitivebehavioral), and educational. ${ }^{[32]}$ These interventions should involve both the patients and their families, caregivers which include staff in residential setting, with the aim to promote an appropriate manifestation of sexual behavior rather than complete eradication of it. The rationale for allowing appropriate expression of sexual behavior is that sexuality is part of human nature throughout life, both physiologically and emotionally. ${ }^{[33]}$

Environmental approaches include switching the gender of caregiver, avoiding sexually stimulating television or radio programs or magazines with nudity. ${ }^{[33]}$ In nursing homes setting, single rooms and provision for conjugal visits may reduce the frequency of ISB by satisfying the residents' physiological needs. ${ }^{[33]}$

Behavioral approaches include consistent redirection and enhanced communication. ${ }^{[34]}$ Other authors have suggested sensitive explanation to the person with dementia on why such behavior is unacceptable, as well as verbal or physical redirection, are beneficial. However, in cases of moderate to severe dementia, the persons with dementia may not have the cognitive abilities to fully comprehend or retain the explanations given. In institutions, when a resident enters the bedroom of another resident, simply redirecting the person to their room may be sufficient, since this conduct could arise from a search for intimacy without sexual purposes, or disorientation. ${ }^{[33]}$

Cognitive-behavioral therapy (CBT) for ISB aim to re-educate the person with dementia about social norms, encouragement to explore the intentions behind each behavior in order to address cognitive distortions, and negative conditioning techniques; however, this approach can be challenging in people with significant cognitive impairment. ${ }^{[35]}$ CBT is a language based therapy, and patients with significant cognitive impairment have language difficulties, and hence, it may not work. Psycho-education/dementia counselling and support to family, caregiver and staff is equally important.

\section{Pharmacological Management}

Pharmacological therapy should be the last resort in the management of ISB in dementia in view of its potential harm. Its benefits and risks must be carefully balanced, and next of kin informed of the decisions, 
especially when medications are prescribed for their off-label indications. Once decided on initiating medication, the clinician should start at the lowest dose and titrate up slowly, and medication prescription should be tailored individually since there are no agreed guidelines for pharmacological treatment for ISB in dementia.

There have been no well-designed studies to demonstrate the efficacy of pharmacologic management of ISB in patients with dementia. ${ }^{[36,37]}$ There are small studies and/or case reports that showed variable success with antidepressants, anticonvulsant as mood stabilizers, hormonal manipulations (antiandrogens, estrogens, gonadotropin-releasing hormone analogs), antipsychotics, cholinesterase inhibitors, and cimetidine which is a histamine $\mathrm{H} 2$ receptor antagonist. ${ }^{[36,37]}$ However none of these are licensed to treat ISB, hence use of these agents would be strictly off-label. Some of the frequently used drugs in the treatment of ISB are discussed below.

\section{Antidepressants}

The concept of using antidepressants for the treatment of ISB derives from their known antilibidinal effects, ${ }^{\left[{ }^{38]}\right.}$ as well as their anti-obsessional

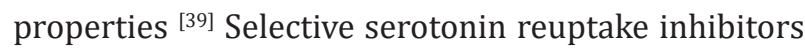
(such as Escitalopram, Fluvoxamine, Paroxetine) are commonly used as first-line agents due to their safety and tolerability profile in the elderly. ${ }^{[40]}$ Based on the case report cited in a review paper by Giorgi RD et al, if a patient fails to respond to SSRI, a second SSRI may be tried. ${ }^{[32]}$ Other antidepressants which may be used include Mirtazapine, ${ }^{[34]}$ TCA or Trazodone. ${ }^{[41,42]}$ However, use of tricyclic antidepressant drugs is generally discouraged due to anticholinergic and cardiovascular adverse effects. ${ }^{[32]}$

Both of the cases presented received SSRI. Mr. A was started on fluvoxamine for his ISB and also to improve sleep at night as Fluvoxamine is sedating. As for Mr. Y he was given Escitalopram to treat his agitation and ISB. Both the patients tolerated SSRIs well with no adverse events reported.

\section{Antipsychotics}

A number of RCTs have evaluated antipsychotic drugs to treat behavioral symptoms in dementia, but none of these trials focused specifically on sexual behavior. ${ }^{[43]}$ It is commonly use in managing BPSD symptoms, however it is associated with definite risk of cardiac event and stroke in patients with dementia. Nonetheless, case reports have described treatment of ISB with low doses of haloperidol, ${ }^{[44]}$ and the atypical antipsychotic quetiapine. ${ }^{[45,46]}$ Sevda Sarikaya reported a first case of AD with ISB treated successfully with Aripriprazole. ${ }^{[47]}$

\section{Anticonvulsants}

Two anticonvulsants are cited to have been shown to be effective in case reports, i.e. Carbamazepine and Gabapentin. ${ }^{[32]}$ Gabapentin is known to cause reduced libido, anorgasmia, and erectile dysfunction. [43] Prescribing Carbamazepine for new patients of Asian ancestry requires prior genotyping for HLA-B*1502 in Singapore. This is considered the standard of care regarding Carbamazepine use in never-users of Asian in ethnicity. ${ }^{[48]}$

\section{CHOLINESTERASE INHIBITORS}

Cholinesterase inhibitors may affect sexual function in many ways, including altering testosterone levels, but the exact mechanisms involved are still uncertain. ${ }^{[49]}$

\section{Beta-Blockers}

It can cause sexual dysfunction and reduced sexual behavior, possibly via a decrease of the adrenergic drive. ${ }^{[50]}$

\section{FINASTERIDE}

Is a $5 \alpha$-reductase inhibitor that blocks conversion of testosterone to dihydrotesterone. It is one of the drugs used in treating benign prostatic hyperplasia. Its potential side effect includes impotence, decrease libido and erectile dysfunction. A case series described successful use of finasteride to treat ISB in 11 elderly men with vascular dementia, with disappearance of inappropriate sexual behavior in 6 of the 11 men within 8 weeks of treatment. ${ }^{[51]}$

\section{HORMONAL MANiPULATIONS}

Lothstein et al described successful use of estrogen to manage sexual disinhibition in 39 elderly patients who had not responded to treatment with SSRIs (level III evidence), hence propose a treatmentalgorithm for ISB that begins with SSRIs and moves to either estrogen or antiandrogen treatment if behavior does not respond to SSRIs. ${ }^{[52]}$ Another case report described using 1 mg of the synthetic estrogen diethylstilbestrol daily to reduce sexual aggression displayed by an elderly man with dementia (level III evidence). ${ }^{[53]}$ Estrogens decrease secretion of luteinizing hormone and follicle- 
stimulating hormone, which lowers testosterone production and typically results in reduced libido.

Medroxyprogesterone (MPA) indirectly decreases the level of testosterone by inhibiting the secretion of pituitary luteinizing hormone (LH) and folliclestimulating hormone (FSH). Cyproterone acetate (CPA) inhibits the interaction between endogenous androgens and androgen receptors, as well as reducing the biosynthesis of androgens. Gonadotropinreleasing hormone analogs (GnRHA) suppress testosterone production by stimulating the secretion of pituitary LH and FSH, with subsequent increase in estrogen levels and decrease of testosterone. Based on the case reports cited in a review paper by Giorgi RD et al, hormonal therapy, with MPA, CPA, estrogen and GnRHA has been used successfully as an alternative treatment after dementia related ISB failed to respond to antipsychotic or sedative medication. ${ }^{[32]}$

Safety and side effects are important issues in prescribing hormonal agents. In females, there may be postmenopausal symptoms, though this may be less common in the older post-menopausal women. Osteoporosis, edema, weight change, and mood changes may occur. Costs of GnRHA can be high, while estrogens and antiandrogens are generally less expensive [32].

\section{COMBINATION THERAPY}

Combination therapy with various medications described above has been suggested for persistent ISB ${ }^{[43]}$ Several case reports describe combinations of drugs to treat these symptoms, ${ }^{[54]}$ however clinicians need to be very careful as combination drugs may cause significant drug-drug interaction especially in the elderly population.

We have used this approach in Mr. A in view of his persistent ISB in the ward so as to potentiate the libido-lowering effect of each drug with improvement of ISB. He was discharged with the following medications: Fluvoxamine, Finasteride, Gabapentin, Atenolol, Donepezil and Olanzapine. Mr. A tolerated all the medications, with no adverse drug reaction noted while inpatient. As for Mr. Y, he was given Escitalopram and added finasteride, which may also improve his BPH symptoms.

\section{CONCLUSION}

Presented are two cases of elderly male dementia patients with inappropriate sexual disinhibition, both managed satisfactorily with non-pharmacological and pharmacological approaches. Although there is no specific guideline in managing ISB, approach must be individualized and should always start with nonpharmacological measures to address the behavior, mainly because pharmacological treatment utilised are all used for off-label indications, and elderly patients are vulnerable for adverse drug reaction. If pharmacologic treatment is warranted, the clinician should always remember to start low and go slow.

Management of ISB can be challenging. Failure to control the symptoms may lead to social isolation resulting in functional decline and institutionalization. A good and thorough assessment, together with education and counselling for the caregivers are important, to plan for management strategies.

\section{REFERENCES}

[1] Lyketsos CG, Lopez O, Jones B, et al. Prevalence of neuropsychiatric symptoms in dementia and mild cognitive impairment: results from the Cardiovascular Health Study. JAMA 2002; 288: 1475-1483.

[2] Rabins PV, Lyketsos CG, Steele C. Practical Dementia Care. New York, NY: Oxford University Press; 1999.

[3] Lyketsos CG, Steele C, Steinberg M. Neuropsychiatric symptoms in dementia. In: Gallo JJ, Busby-Whitehead J, Rabins PV, Silliman R, Murphy J, eds. Reichel's Care of the Elderly: Clinical Aspects of Aging. 5th ed. Baltimore, Md: Williams \& Wilkins; 1999:214-228.

[4] Vaingankar JA, Chong SA, Abdin E, Picco L, Jeyagurunathan A, Seow E, Ng LL, Prince M, Subramaniam M. Behavioral and psychological symptoms of dementia: prevalence, symptom groups and their correlates in communitybased older adults with dementia in Singapore. International psychogeriatrics. 2017 Aug; 29(8):1363-76

[5] Steinberg M, Shao H, Zandi P, et al. Point and 5 -year period prevalence of neuropsychiatric symptoms in dementia: the Cache County Study. Int J Geriatr Psychiatry 2008; 23: 170-177

[6] ega MS, Cummings JL, Fiorello T, et al. The spectrum of behavioral changes in Alzheimer's disease. Neurology 1996; 46: 130-135. 
[7] Series H, Dégano P. Hypersexuality in dementia. Adv in Psych Treat. 2005; 11:424-31.

[8] Alagiakrishnan K, Lim D, Brahim A, Wong A, Wood A, Senthilselvan A, et al. Sexually inappropriate behaviour in demented elderly people. Postgrad Med J. 2005;81(957):463-6.

[9] Szasz G. Sexual incidents in an extended care unit for aged men. J Am Geriatr Soc. 1983; 31 (7): 407-11.

[10] Burns A, Jacoby R, Levy R. Psychiatric phenomena in Alzheimer's disease. IV: disorders of behaviour. Brit J Psychol. 1990;157:86-94.

[11] Kamel HK, Hajjar RR. Sexuality in the nursing home, part 2: managing abnormal behavior-legal and ethical issues. J Am Med Dir Assoc. 2004;5(2 Suppl):S48-52.

[12] Bird, M., Moniz-Cook, E. Challenging behavior in dementia: a psychosocial approach to intervention. Handbook of the clinical psychology of ageing, $p$ 443. (2008)

[13] Wallace M, Safer M. Hypersexuality among cognitively impaired older adults. Geriatr Nurs. 2009;30(4):230-7.

[14] Alkhalil, C., Tanvir, F., Alkhalil, B., Lowenthal, D.T.: Treatment ofsexualdisinhibitionindementia:case reports and review of the literature. Am. J. Ther. 11(3), 231-235 (2004)

[15] Fabà, J. \& Villar, F. (2013, fevereiro). Dementia and inappropriate sexual behavior (ISB): What we know and what we need to know. Revista Temática Kairós Gerontologia,16(1), ?]Eroticism/ Sexuality and Old Age

[16] Johnson C, Knight C, Alderman N. Challenges associated with the definition and assessment of inappropriate sexual behaviour amongst individuals with an acquired neurological impairment. Brain Inj. 2006;20(7):687-93.", pp.49-68.

[17] De Medeiros K, Rosenberg PB, Baker AS, Onyike CU. Improper sexual behaviors in elders with dementia living in residential care. Dement Geriatr Cogn Disord. 2008;26(4):370-7.

[18] Levitsky AM, Owens NJ. Pharmacologic treatment of hypersexuality and paraphilias in nursing home residents. J Am Geriatr Soc. 1999;47(2):231-4.
[19] Onishi J, Suzuki Y, Umegaki H, Endo H, Kawamura $\mathrm{T}$, Imaizumi M, et al. Behavioral, psychological and physical symptoms in group homes for older adults with dementia. Int Psychogeriatr. 2006;18(1):75-86.

[20] Robinson, K.M. (2003). Understanding hypersexuality: A behavioral disorder of dementia. Home Healthcare Nurse, 21(1), 43-47.

[21] Zeiss A.M., Davies H.D. \& Tinklenberg J.R. (1996) An observational study of sexualbehavior in demented male patients. Journal of Gerontology 51, M325-M329.

[22] Knight C., Alderman N., Johnson C., et al. (2008) The St Andrews Sexual BehaviourAssessment (SASBA): development of a stan-dardised recording instrument for the assessment and measurement of challenging sexual behaviour in people with progressive and acquired neurological impairment. Neuropsychological Rehabilitation 18,129-159.

[23] Onishi, J., Suzuki, Y., Umegaki, H., Endo, H., Kawamura, T., Imaizumi, M. \& Iguchi, A. (2006). Behavioral, psychological and physical symptoms in group homes for older adults with dementia. International Psychogeriatrics, 18(1), 75-86.

[24] Mattison, A. \& Hemberg, M. (1998). Intimacymeeting needs and respecting privacy in the care of elderly people: What is a good moral attitude on the part of the nurse/carer? Nursing Ethics: An International Journal for Health Care Professionals, 5(6), 527-534.

[25] Mayers, K.S. (1998). Sexuality in the demented patient. Sexuality and Disability, 16(3), 219-225.

[26] Haddad, P.M. \& Benbow, S.M. (1993). Sexual problems associated with dementia: Part 1. Problems and their consequences. International Journal of Geriatric Psychiatry, 8(7), 547-551.

[27] Kafka MP, Prentky R. A comparative study of nonparaphilic sexual addictions and paraphilias in men. J Clin Psychol. 1992;53(10):345-50.

[28] Paton C. Benzodiazepines and disinhibition: a review. Psychiatric Bulletin. 2002 Dec; 26 (12):460-2.

[29] Van Gaalen MM, Brueggeman RJ, Bronius PF, Schoffelmeer AN, Vanderschuren LJ. Behavioral 
Managing Sexually Inappropriate Behaviour among the Older Adults with Dementia

disinhibition requires dopamine receptor activation. Psychopharmacology. 2006 Jul 1;187(1):73-85.

[30] Ambar G, Chiavegatto S. Anabolic-androgenic steroid treatment induces behavioral disinhibition and downregulation of serotonin receptor messenger RNA in the prefrontal cortex and amygdala of male mice. Genes, Brain and Behavior. 2009 Mar;8(2):161-73

[31] Tucker I. Management of inappropriate sexual behaviors in dementia: a literature review. Int Psychogeriatr. 2010;22(5):683-92.

[32] De Giorgi R, Series H. Treatment of inappropriate sexual behavior in dementia. Current treatment options in neurology. 2016 Sep 1;18(9):41.

[33] Ibrahim C, Reynaert C. Hypersexuality in neurocognitive disorders in elderly people-a comprehensive review of the literature and case study. Psychiatr Danub. 2014; 26 (Suppl 1): 36-40.

[34] Bardell A, Lau T, Fedoroff JP. Inappropriate sexual behavior in a geriatric population. Int Psychogeriatr. 2011;23(7):1182-8.

[35] Derouesné C. The so-called hypersexual behaviors in dementia. Psych Neuropsychiatr Vieil. 2009;7(2):101-8.

[36] Ozkan B, Wilkins K, Muralee S, Tampi RR. Pharmacotherapy for inappropriate sexual behaviors in dementia: a systematic review of literature. Am J Alzheimers Dis Other Demen. 2008;23(4):344-354.

[37] Black B, Muralee S, Tampi, RR. Inappropriate sexual behaviors in dementia. J Geriatr Psychiatry Neurol. 2005;18(3):155-162

[38] Montejo AL, Montejo L, Navarro-Cremades F. Sexual side-effects of antidepressant and antipsychotic drugs. Curr Opin Psychiatry. 2015;28(6):418-23.

[39] Pizarro M, Fontenelle LF, Paravidino DC, Yücel M, Miguel EC, de Menezes GB. An updated review of antidepressants with marked serotonergic effects in obsessive-compulsive disorder. Exp Opin Pharmacother. 2014;15(10):1391-401.

[40] Wilson K, Mottram P. A comparison of side effects of selective serotonin reuptake inhibitors and tricyclic antidepressants in older depressed patients: a meta-analysis. Int J Geriatr Psychiatry. 2004;19(8):754-62.

[41] Leo RJ, Kim KY. Clomipramine treatment of paraphilias in elderly demented patients. J Geriatr Psychiatry Neurol 1995;8(2):123-4

[42] Simpson DM, Foster D. Improvement in organically disturbed behavior with trazodone treatment. J Clin Psychiatry 1986;47(4):191-3.

[43] Joller P, Gupta N, Seitz DP, Frank C, Gibson M, Gill SS. Approach to inappropriate sexual behaviour in people with dementia. Can Fam Physician. 2013;59(3):255-60.

[44] Kobayashi T. Effect of haloperidol on a patient with hypersexuality following frontal lobe injury. Psychogeriatrics. 2004;4(2):49-52.

[45] MacKnight C, Rojas-Fernandez C. Quetiapine for sexually inappropriate behavior in dementia. J Am Geriatr Soc. 2000;48(6):707.

[46] Prakash R, PathakA, Munda S, Bagati D. Quetiapine effective in treatment of inappropriate sexual behavior of Lewy body disease with predominant frontal lobe signs. Am J Alzheimers Dis Other Demen. 2009;24(2):136-40. Epub 2009 Jan 7.

[47] Sarikaya S, Sarikaya B. Aripiprazole for the Treatment of Inappropriate Sexual Behavior: Case Report of an Alzheimer's Disease Patient Known as Heterosexual with Recently Shifted Sexual Orientation to Same Gender. Journal of Alzheimer's Disease Reports. 2018

[48] https://www.hsa.gov.sg/content/dam/HSA/ HPRG/Safety_Alerts_Product_Recalls_Enforcement/ Severe\%20Cutaneous\%20Adverse\%20Reactions\%20 (SCAR)\%20Guide_WBv2.pdf

[49] Haywood WM, Mukaetova-Ladinska EB. Sex influences on cholinesterase inhibitor treatment in elderly individuals with Alzheimer's disease. Am J Ger Pharmacother. 2006;4(3):273-86

[50] La Torre A, Giupponi G, Duffy D, Conca A, Catanzariti D. Sexual dysfunction related to drugs: a critical review. Part IV: cardiovascular drugs. Pharmacopsychiatry. 2015;48(1):1-6.

[51] Na HR, Lee JW, Park SM, Ko SB, Kim S, Cho ST. Inappropriate sexual behaviors in 
Managing Sexually Inappropriate Behaviour among the Older Adults with Dementia

patients with vascular dementia: possible response to finasteride. J Am Geriatr Soc. 2009;57(11):2161-2.

[52] Lothstein LM, Fogg-Waberski J, Reynolds P. Risk management and treatment of sexual disinhibition in geriatric patients. Conn Med. 1997;61(9):609-18.
[53] Kyomen HH, Nobel KW, Wei JY. The use of estrogen to decrease aggressive physical behavior in elderly men with dementia. J Am Geriatr Soc. 1991;39(11):1110-2.

[54] Guay DR. Inappropriate sexual behaviors in cognitively impaired older individuals. Am J Geriatr Pharmacother. 2008;6(5):269-88.

Citation: Rachelle Tan-Patanao, Eden XJ Tay, WT Chang, SC Lim. Managing Sexually Inappropriate Behaviour among the Older Adults with Dementia. Open Journal of Geriatrics. 2019; 2(2): 23-31.

Copyright: (C) 2019 Rachelle Tan-Patanao, Eden XJ Tay, WT Chang, SC Lim. This is an open access article distributed under the Creative Commons Attribution License, which permits unrestricted use, distribution, and reproduction in any medium, provided the original work is properly cited. 